\title{
Applicability of Magnetic Resonance Imaging for Early Diagnosis of Common Peroneal Neuropathy
}

\author{
Gwang Yoon Choi, Jinseo Yang, Yongjun Cho, Hyukjai Choi, Jinpyeong Jeon, Sukhyung Kang \\ Department of Neurosurgery, Chuncheon Sacred Heart Hospital, Hallym University College of Medicine, Chuncheon, Republic of Korea
}

Corresponding author:

Jinseo Yang

Department of Neurosurgery,

Chuncheon Sacred Heart Hospital,

Hallym University College of

Medicine, 77 Sakju-ro, Chuncheon

24253, Republic of Korea

Tel: $+82-33-240-5173$

Fax: $+82-70-5096-8691$

E-mail: yiyaseo@naver.com
Received: August 28, 2021

Revised: September 15, 2021

Accepted: September 18, 2021

\begin{abstract}
Objective: This study aimed to assess the clinical applicability of magnetic resonance imaging (MRI) for the early diagnosis of common peroneal neuropathy (CPNe).

Methods: Over three years, the authors have treated 58 patients with CPNe. All patients had clinical or neurophysiological confirmation of CPNe. Among them, 35 (60\%) patients underwent axial knee MRI with a 1.5-Tesla scanner. These 35 patients were selected for study and were classified into three groups according to the time of examination after the occurrence of dropped foot-acute, subacute, and chronic onset groups. According to muscle appearances (normal, edematous change, and atrophy), we diagnosed them with $\mathrm{CPNe}$, except for those with normal morphology. We evaluated the applicability of MRI in the diagnosis of CPNe compared to that of electromyography (EMG).

Results: The 18, 11, and six cases were included in the acute, subacute, and chronic onset groups, respectively. In the acute onset group, three cases had normal muscle appearance, while 15 cases had edematous changes in the affected muscles. In the subacute onset group, eight cases had edematous changes, while three cases showed muscle atrophy. In the chronic onset group, six cases had muscle atrophy. CPNe could be diagnosed using MRI in about $91 \%$ (32/35) of all the cases. Excluding the chronologically chronic stage, diagnosis rate was approximately $89 \%(26 / 29)$ of all the cases. However, only in 27 cases (77\%) denervation potentials were presented on EMG.

Conclusion: According to our results, MRI is a helpful diagnostic modality, especially in the early stage of $\mathrm{CPNe}$, and may lead to proper management.
\end{abstract}

Key Words: Electrophysiology; Magnetic resonance imaging; Peroneal neuropathies

\section{INTRODUCTION}

Common peroneal neuropathy (CPNe) is a usual cause of dropped foot, which results from various causes, including idiopathic causes, iatrogenic causes, tumors, and trauma. Taking detailed patient history and performing neurological examination are the most important initial clinical approaches to reach a diagnosis of suspected $\mathrm{CPNe}$, while final diagnostic confirmation depends on the electrodiagnostic study (EDX) ${ }^{1,7,14)}$.

As neuropraxic lesions rarely show positive electromyographic finding ${ }^{5}$, for EDX, patients have to wait for confirmation of diagnosis until Wallerian degeneration initiates after the onset of symptom; thus, it could take a certain amount of time to establish the diagnosis. In contrast, magnetic resonance imaging (MRI) requires no such delay to find denervation and has played an important role in the detection and characterization of pathological conditions of skeletal muscles that change muscle signal intensity ${ }^{4)}$.

The CPNe innervates the muscles of the anterior and lateral compartments of the lower leg"). Peroneus longus is situated in the lateral compartment and both extensor digitorum longus and tibialis anterior in the anterior compartment ${ }^{3)}$ (Fig. 1). Using conven- tional MRI, CPNe can be diagnosed with signal changes from denervation of these muscles.

For 3 years, the current study's authors performed conventional knee MRl in patients with CPNe and found signal changes in the muscles in the antero-lateral compartment during diagnostic process.

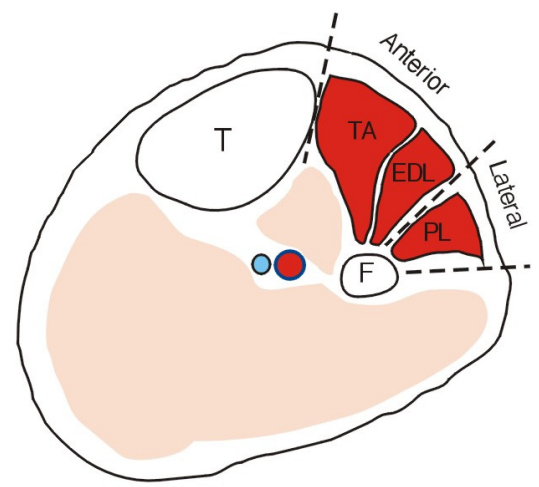

Fig. 1. Schematic description of the muscles and compartments on the right knee for the diagnosis of common peroneal neuropathy. T: tibia; F: fibula; TA: tibialis anterior muscle; EDL: extensor digitorum longus muscle; PL: peroneus longus muscle. 
Table 1. Summary of the MRI findings of muscle denervation in the 3 stages and their corresponding histological features

\begin{tabular}{lccc}
\hline \hline 3 stages & Histological changes of denervated muscles & MRI signal changes within muscle \\
\hline Acute $(<2$ weeks) & Edematous & T2-W & Increased \\
& & STIR & Increased \\
Subacute (2-4 weeks) & Further increase in extracellular water & T2-W & Increased \\
& & STIR & Increased \\
Chronic ( $>1$ month) & Fatty infiltration and atrophy & T1-Wl & Increased \\
& & T2-W & Increased \\
\hline
\end{tabular}

MRI: magnetic resonance imaging; T2-W: T2-weighted image; T1-WI: T1-weighted image; STIR: short tau inversion recovery.

The purpose of this study was to assess the clinical applicability of MRI for the early diagnosis of patients with CPNe.

\section{MATERIALS AND METHODS}

We retrospectively examined medical records of 58 patients diagnosed with CPNe confirmed by clinical approaches or EDX from December 2017 to December 2020. Among these patients, 35 underwent axial MRI with a 1.5-Tesla scanner (Gyroscan Intera; Philips Medical Systems, Best, the Netherlands) from the apex of the popliteal fossa to $20 \mathrm{~cm}$ distal from the fibular head. Only these subjects were included in the study.

Patients were classified into three chronological groups according to the time of examination after the occurrence of dropped footacute, subacute, and chronic onset groups. Patients in the acute onset group underwent examination within the first 1 to 2 weeks of onset, those in the subacute onset group underwent examination within 3 to 4 weeks of onset, and those in the chronic onset group underwent examination after $>4$ weeks of onset.

MRI was performed for all the subjects for this study immediately after the initial clinical evaluation. The muscle findings of MRI were chronologically classified as shown in Table 1. Short tau inversion recovery (STIR) images are highly water-sensitive, and the timing of the pulse sequence used suppresses signals coming from fatty tissues; therefore, only water molecules are visualized bright. The acutely and subacutely denervated muscle shows a high-signal intensity pattern on fluid-sensitive sequences and normal signal intensity on Tl-weighted MRI because of muscle edema. In chronic denervation, muscle atrophy and fatty infiltration demonstrate high signal changes on $\mathrm{Tl}$-weighted sequences association with volume loss. Effectively applicable ranges of muscle volume to define atrophy, of muscle/fat infiltration ratio to define atrophy, and of the degree of signal intensity to define muscular edema were not available in other literatures. This problem could have been solved if we compared the MRI of the affected side to that of the unaffected side, but we could not perform the MRls of both knees due to the longer time required and the financial reason. Therefore, we depended upon the radiologist's reading and the double-checking from 2 of the authors (C.G.Y. and Y.J.S.).

All patients underwent EDX 4 weeks after onset. If the neuropathy was chronic, the patient underwent EDX immediately. The result
Table 2. Demographic data of all patients (35 cases)

\begin{tabular}{lc}
\hline \hline Variables & Value $(\mathrm{n}=35)$ \\
\hline Mean age (range) & $49.3(25-76)$ \\
Sex & \\
Male & $21(60.0)$ \\
Female & $14(40.0)$ \\
Causes & \\
Idiopathic & $32(91.4)$ \\
Trauma & $3(8.6)$ \\
Affected side & \\
Right & $19(54.3)$ \\
Left & $16(45.7)$ \\
Duration of symptoms & \\
Acute & $18(51.4)$ \\
Subacute & $11(31.4)$ \\
Chronic & $6(17.1)$ \\
\hline
\end{tabular}

of delayed nerve conduction velocity (NCV) was subdivided into muscle denervation and without. The study was approved by Chuncheon Sacred Heart Hospital Institutional Review Board.

\section{RESULTS}

\section{Baseline Characteristics}

Table 2 represents the demographic data of the patients. The sample showed 2:1 ratio between male and female. The patients' age ranged from 25 to 76 years, and the mean age was 49.3 years. The etiologies of CPNe were idiopathic in 32 patients (91.4\%) and traumatic in 3 patients (8.6\%). In 3 patients with traumatic CPNe, there was no direct injury to the musculature related to the CPN. The modes of trauma were fibular fracture in 2 patients and laceration of the knee in 1 patient. We could not find a significant difference in the affected laterality. The chronicity of the CPNe was: acute in $18(51.4 \%)$; subacute in $11(31.4 \%)$; chronic in $6(17.1 \%)$ patients. 

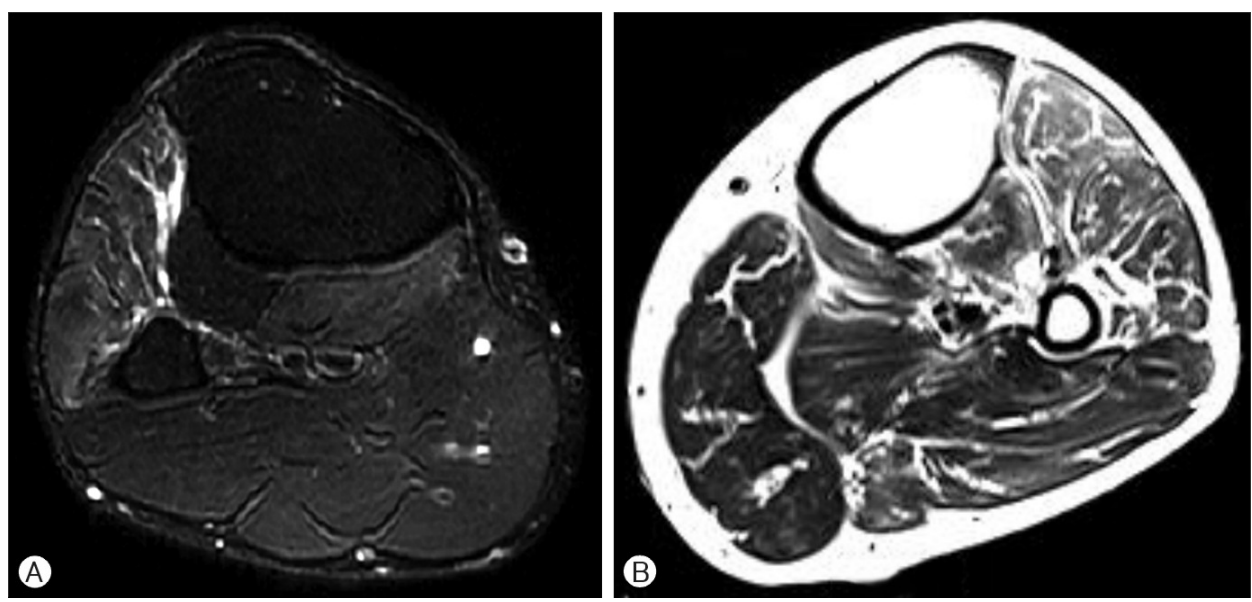

Fig. 2. (A) Axial fat-saturated T2-weighted magnetic resonance imaging (MRI) (TR, $3500 \mathrm{~ms}$; TE, $65 \mathrm{~ms}$ ) at the level of the proximal entrance to the fibular head demonstrating edematous high signal changes within muscles of the anterolateral compartment of the lower extremity. (B) Axial T1-weighted MRI (TR, $150 \mathrm{~ms}$; TE $4800 \mathrm{~ms}$ ) showing fatty infiltration and atrophy of the affected muscles in the anterolateral compartment.

Table 3. MRI appearances of affected muscles

\begin{tabular}{lcl}
\hline \hline Stages & Cases & MRI appearance \\
\hline Acute & 3 & Normal \\
& 15 & Edematous \\
Subacute & 8 & Edematous \\
& 3 & Atrophy \\
Chronic & 6 & Atrophy \\
\hline
\end{tabular}

MRI: magnetic resonance imaging.

\section{MRI Appearances of the Muscles}

MRI showed positive findings in 32 cases. The classified findings are summarized in Table 3. The affected muscles were: multiple muscles in the anterolateral compartment simultaneously in 30 cases; single muscle (peroneus longus) in two cases. CPNe could be diagnosed using MRI in about 91\% (32/35) of all the cases. Excluding the chronologically chronic stage, the diagnosis rate was approximately $90 \%(26 / 29)$ of all the cases. The MRI changes in the affected muscle included a high-signal intensity in fluid-sensitive sequences (T2-weighted and STIR images) in 23 cases and a high-signal intensity of fat infiltration and atrophy with decreased muscle volume (TT and T2-weighted images) in 9 cases.

\section{EDX Results}

All the patients showed abnormality in the NCS, but in 8 patients, normal electromyography (EMG) was obtained. In the acute onset group, eight cases had delayed latency of NCV with normal EMG. In the remaining 27 cases (77\%), positive sharp waves and acute fibrillation were noted, confirming muscle denervation.

\section{DISCUSSION}

The common peroneal nerve (CPN), a branch from the sciatic nerve, descends obliquely along the lateral side of the popliteal fossa to the head of the fibula and then winds around the neck of the fibula. The CPN enters the anterior and lateral muscle compartments of the leg and gives branches. These include superficial peroneal nerve, which innervates peroneus longus and brevis for ankle eversion; deep peroneal nerve, which innervates the tibialis anterior for ankle dorsiflexion as well as the extensor digitorum longus and brevis for toe extension ${ }^{1,7,13,14)}$. We thought the connection between the CPN and the muscles might lead to the diagnostic potential of MRI by identifying alterations in muscles.

During muscle denervation, muscle blood content increases as the intramuscular capillary bed get proliferated. Also, extracellular fluid within the denervated muscle cells increases, probably due to increased cell membrane permeability and reduced Na-K-ATPase function $^{2,8,10)}$. Therefore, abnormalities are seen in the denervated muscles on water-sensitive MRI sequences ${ }^{4,6,9,12)}$. Peripheral nerve injury also induces metabolic changes. Glucose metabolism takes place in the way that new glucose uptake is inhibited while glycolysis is activated. Furthermore, proteolysis increases ${ }^{10}$. These metabolic changes result in morphological change, shown as atrophy. This phenomenon occurs as soon as the nerve gets damaged and can also be demonstrated histologically as a reduction in the muscle fiber diameter. As atrophy progresses, the ratio of fat to protein tends to increase $2,6,8,10$. Therefore, we can see the shrinkage of muscle bulk and the fatty infiltration within the muscle in chronic denervation. In experimental studies, moderate atrophy was seen as early as approximately 10 to 12 days after denenvation. However, it typically takes 3 weeks after the insult to the nerve to observe pat- 
hognomonic findings histologically ${ }^{8}$.

Several clinical and experimental studies have demonstrated signal

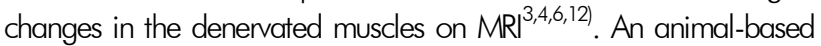
study demonstrated that changes could be observed on MRI as early as within as $24 \mathrm{hr}$ after injury ${ }^{6}$. In a clinical study, an abnormality from denervation could be demonstrated within 4 days of injury ${ }^{12}$. In this study, we performed a conventional axial MRI of the knee, which revealed the degree of muscle denervation according to its time course. In three cases, however, we could not find any signal changes in the muscles although we performed MRI 3 to 5 days after the onset. This result did not match the results of the aforementioned studies.

Acute, subacute, and chronic stages were defined by shortening the generally used period classification to evaluate the usefulness of early diagnosis. According to our results, early diagnosis before chronic denervation was possible in $74 \%(26 / 35)$ cases. This fact, despite the lack of adequate sample size, calls for the need to further study the usefulness of MRI.

EDX changes occur within the affected muscles after denervation, which can be demonstrated on $E M G^{7}$. It has been shown experimentally that early detection of spontaneous activity of the affected muscle is possible. However, the EMG changes are considered to occur late, justifying the clinicians to delay the exam for at least 2 weeks from the injury ${ }^{4,6}$. In addition, EDX is generally known for its invasiveness ${ }^{4,6,11)}$. In our study, all subjects showed abnormal EDX findings, and 27 (77\%) patients had muscle denervation. The detection rate of denervation was similar to the early diagnosis rate of MRI.

We would like to briefly present 2 cases demonstrating the usefulness of MRI in the diagnosis of CPNe. A 52-year-old male patient presented to our clinic with a chief complaint of right-sided progressive dropped foot 2 weeks prior to consultation. On admission, a positive Tinel sign was found on the fibular neck, and neurologic examination revealed ankle dorsiflexion Grade 3/5, big toe dorsiflexion Grade 3/5, ankle eversion Grade 4/5, and ankle inversion Grade 5/5. MRI showed muscle swelling of the anterolateral compartment without atrophy (Fig. 2A). Given these results, the diagnosis of right-sided CPNe was made, and the patient was advised to wait for spontaneous recovery. After 1 month, EMG revealed muscle denervation compatible with CPNe. In this case, we were able to make an early diagnosis based on both MRI finding and clinical manifestation prior to EDX, due to the required waiting time for detection.

In another case, a 55-year-old male patient visited our clinic with sudden onset of left-sided dropped foot 3 months prior to consultation. On admission, neurological examination revealed ankle dorsiflexion Grade 1/5, big toe dorsiflexion Grade 2/5, ankle eversion Grade 2/5, and ankle inversion Grade 5/5. EMG showed muscle denervation related to the CPN, and MRI revealed fatty infiltration with atrophy of the antero-lateral compartment of the lower extremity (Fig. 2B). We diagnosed the patient with left-sided $\mathrm{CPNe}$ and performed external neurolysis and decompression via the fibular tunnel approach. At the 1-month follow-up after surgery, there was an improvement in muscle strength. In this case, we could observe the EMG findings of denervation atrophy were well portrayed in the MRI as fattly infiltration in the muscle and the shrinkage of muscle volume.

Both EMG and MRI can diagnose muscle denervation and document the time course of muscle atrophy ${ }^{4,6,11}$. However, considering the invasiveness and time-consuming aspect of EDX, and as MRI can portray the electrophysiological and histological changes after denervation, MRI can be an effective tool for localizing and defining the extent of $\mathrm{CPNe}^{4,6,9,12)}$.

This study has some limitations. First, as a retrospective study from a single institution, there was potential for several biases. Second, we enrolled a small number of patients; thus, the results of our study cannot be generalized.

\section{CONCLUSION}

According to our results, MRI is a useful diagnostic modality for detecting and assessing CPNe in the early stage of management.

\section{CONFLICTS OF INTEREST}

No potential conflict of interest relevant to this article was reported.

\section{REFERENCES}

1. Baima J, Krivickas L: Evaluation and treatment of peroneal neuropathy. Curr Rev Musculoskelet Med 1:147-153, 2008

2. Clausen $\mathrm{T}$ : Regulation of active $\mathrm{Na}^{+}-\mathrm{K}^{+}$transport in skeletal muscle. Physiol Rev 66:542-580, 1986

3. Donovan A, Rosenberg ZS, Cavalcanti CF: MR imaging of entrapment neuropathies of the lower extremity. Part 2. The knee, leg, ankle, and foot. Radiographics 30:1001-1019, 2010

4. Kamath S, Venkatanarasimha N, Walsh M, Hughes P: MRI appearance of muscle denervation. Skeletal Radiology 37:397404, 2008

5. Kamble N, Shukla D, Bhat D: Peripheral nerve injuries: Electrophysiology for the neurosurgeon. Neurology India 67:14191422, 2019

6. Kikuchi Y, Nakamura T, Takayama S, Horiuchi Y, Toyama Y: MR imaging in the diagnosis of denervated and reinnervated skeletal muscles: Experimental study in rats. Radiology 229: 861-867, 2003

7. Masakado Y, Kawakami M, Suzuki K, Abe L, Ota T, Kimura A: Clinical neurophysiology in the diagnosis of peroneal nerve palsy. Keio J Med 57:84-89, 2008

8. Sarnat HB, Portnoy JM, Chi DY: Effects of denervation and tenotomy on the gastrocnemius muscle in the frog: A histologic and histochemical study. Anat Rec 187:335-346, 1977

9. Shabas D, Gerard G, Rossi D: Magnetic resonance imaging examination of denervated muscle. Comput Radiol 11:9-13, 1987

10. Shoji S: Effect of denervation on glucose uptake in rat soleus and extensor digitorum longus muscles. Muscle Nerve 9:6972, 1986 
11. Viddeleer AR, Sijens PE, van Ooyen PM, Kuypers PD, Hovius SE, Oudkerk M: Sequential MR imaging of denervated and reinnervated skeletal muscle as correlated to functional outcome. Radiology 264:522-530, 2012

12. West GA, Haynor DR, Goodkin R, Tsuruda JS, Bronstein AD, Kraft G, et al.: Magnetic resonance imaging signal changes in denervated muscles after peripheral nerve injury. Neurosurgery 35:1077-1085, 1994
13. Yang J, Cho Y, Cho J, Choi H, Jeon J, Kang S: Anatomical variants of "Short Head of Biceps Femoris Muscle" associated with common peroneal neuropathy in Korean populations: An MRI based study. J Korean Neurosurg Soc 61:509-515, 2018

14. Yu JK, Yang JS, Kang SH, Cho YJ: Clinical characteristics of peroneal nerve palsy by posture. J Korean Neurosurg Soc 53: 269-273, 2013 